\title{
Electric Superbike Racing - the Design and Construction of a Championship Winning Electric Superbike
}

\author{
Patrick Wheeler \\ Power Electronics, Machines and \\ Control Research Group, \\ University of Nottingham, UK \\ Pat.wheeler@nottingham.ac.uk
}

\author{
Jonathan Blissett \\ Power Electronics, Machines and \\ Control Research Group, \\ University of Nottingham, UK \\ Jonathan.blissett@nottingham.ac.uk
}

\author{
Miquel Gimeno Fabra \\ Power Electronics, Machines and \\ Control Research Group, \\ University of Nottingham, UK \\ M.Gimeno-Fabra@nottingham.ac.uk
}

\begin{abstract}
The electric superbikes recently developed at The University of Nottingham have to be considered as a high performance vehicles, competing with its internal combustion engine driven counterparts. This paper presents the motivations for this work as well the design decisions taken to maximize performance in race conditions, along with modelling undertaken to gain an insight into the system interaction as a whole. Data recorded during races and on a rolling road are presented to validate the simulation work.
\end{abstract}

\section{INTRODUCTION}

This paper will describe some of the design criteria for an electric superbike which has shown that fantastic performance is possible with an electric vehicle. The electric superbike provides a demanding application where the electrical machine and power converter design must be optimised in terms of performance and weight.

There are already a number of electric motorcycles on the market which are used both in racing and private transport, some bespoke designs and others originating from dedicated manufacturers [1] [2] [3]. Their performance in racing is often mixed due to different design targets. Racing has often been used to promote the development of various vehicles utilising new technologies as it gives a chance to test ideas in a demanding environment and presents the technology to a wide audience. Racing also gives the competitive aspect which provides a mechanism to enhance and fund such technological development.

\section{Motivation}

Racing is also exciting and this aspect of the competition is great to dispel myths about the limitation of certain technologies as well as promoting the technology with a gaol of high rates of acceptance. As we move to more and all electrically propelled vehicles this promotion and sense of fun in the technology is going to be an important aspect. Tesla has recently shown how successful this approach can be for cars, bikes such as the one form the University of Nottingham, as shown in Fig. 1, have the capability of doing the same for motorbikes.
Another important aspect of motorsport in the development of any vehicle is that it allows a time when more design risks can be taken. A race vehicle is not expected to have the same reliability as a family car or a truck. Motorsport is therefore a great chance to push the boundaries of the technology in pursuit of maximum performance and a place to try new ideas.

Motorsport can also help in changing perceptions, not only of users, but also of legislators. Examples include the taxation of electric vehicles and the transportation of the Lithium Ion cells commonly used to make the batteries for electric transportation systems (as well as many other applications including mobile phones)..

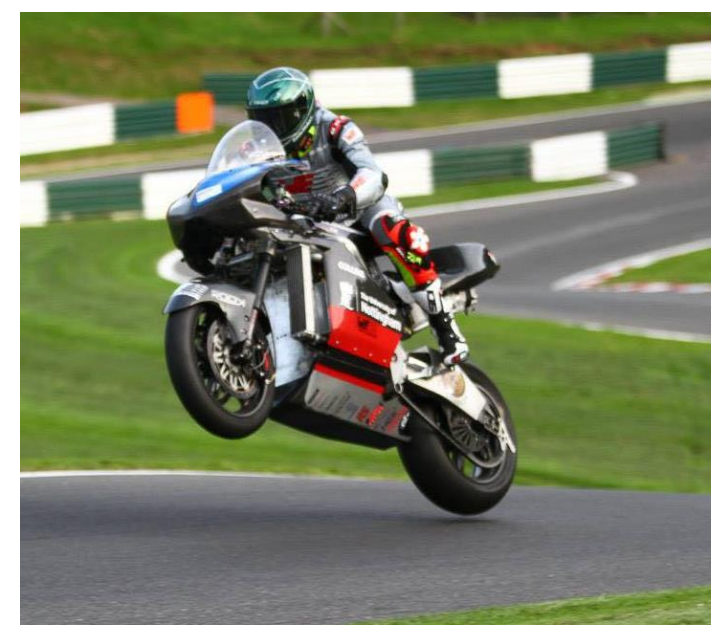

Fig. 1. The University of Nottingham Electric Superbike during Testing in the UK, 2017

\section{THE DRIVETRAIN}

For most electric vehicles, the electric powertrain consists of three main parts - a battery pack, motor drive and motor, as shown in Fig. 2. These are optimized as a system in order to meet the required performance and specific requirements imposed by the vehicle's application. In the case of an electric motorbike it is the complete system weight and to a certain extent volume which must be considered. 
A heavier bike will require more energy for acceleration, but the additional stored energy may meet this requirement as well as extending the top speed and/or range. The efficiency of the motor and drive can also be an important factor, though this is a trade-off because of the weight distribution between these components and the batteries.

Due to the long time-scale needed for a fully optimized design of all the electrical components the bike's powertrain is initially specified using readily available technology, sized by comparing to the specifications and performance of existing sport motorcycles.

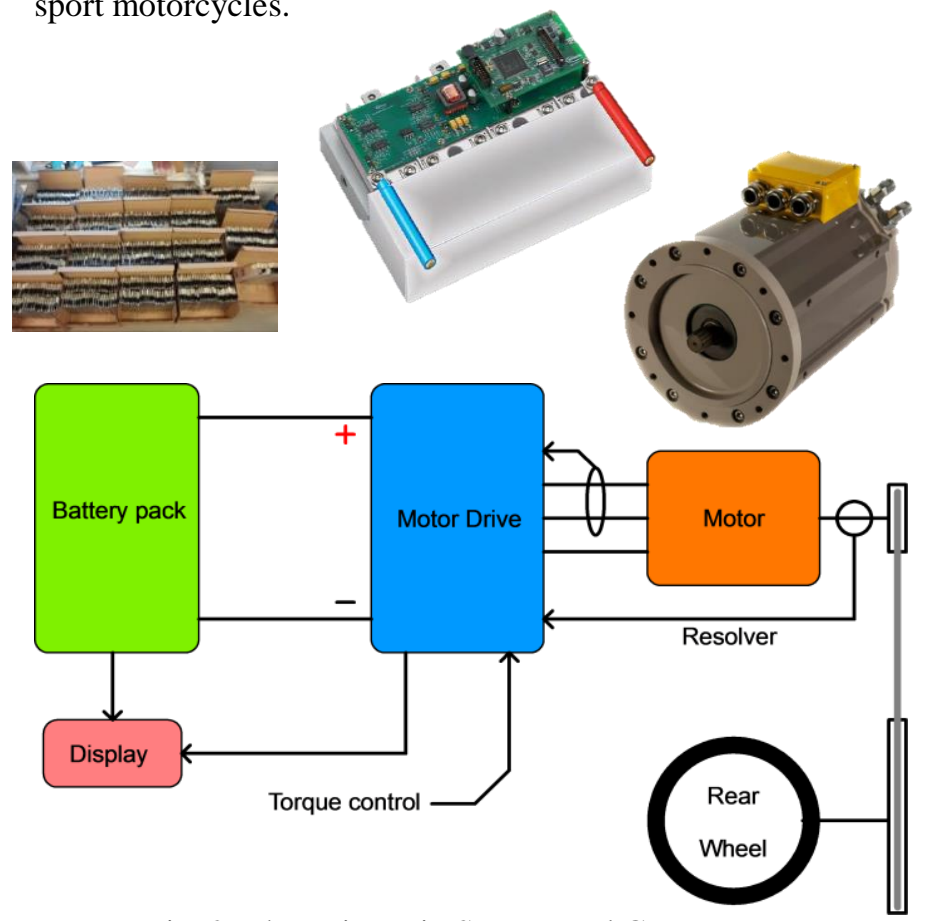

Fig. 2. The Drivetrain System and Components

\section{A. Motor Selection and gearing}

It is well known that for a given power rating, the volume and therefore weight of an electric motor is reduced proportionately. This combined with the desire to reduce unsprung mass on the vehicle suspension [4] dictates using a high speed motor, coupled to the rear wheel via some mechanical reduction gearing. Available traction motors have been compared, considering their weight and efficiency, to find the most suitable machine from existing technology. This comparison showed that a Parker Hannaffin permanent magnet motor [5] was the most suitable.

Due to the lack of accurate, available drive-cycle data for typical race circuits during the early stages of the project, the motor was oversized to give the opportunity for experimentation with different torque-speed characteristics using the same motor. The chosen motors run with a maximum speed of $10500 \mathrm{rpm}$, which when the $2 \mathrm{~m}$ circumference of a typical sport bike tyre is considered, clearly necessitates a mechanical reduction to give a realistic top speed at this 10500rpm maximum motor speed rating.

Many bikes achieve this reductions this using a fixed ratio gearbox, with further reduction achieved using the chain and sprockets. Belt drives are also commonly employed for this purpose in motorcycles, however chain drives are more suited for racing due to the higher torque transmission density they exhibit.

Within the practical diameters of sprockets, it is feasible to couple the chosen motor to the wheel just using a chain as a single reduction mechanism. This options has been compared to other designs in terms of efficiency and weight, by considering chain drives with ratios in the region of interest versus the combined efficiency of a gear and chain drive, for the same transmitted power.

The following chain loss model uses the principle described in [6], where the losses are derived from two sources collisions between the chain roller and sprocket teeth and the work done from the articulation of the chain through the angles required to follow the trajectory upon the sprockets, prescribed by the number of teeth chosen for each sprocket and their centre distance. This allows the chain efficiency to be calculated as a function of torque and speed, with the chain dimensions, sprockets sizes and centre distances as parameters in the model. The analysis in [6] shows that at the expected operating point, collision losses are sufficiently small to neglect, so only the articulation is considered here. The losses depend on the chain tension and the chain articulation angle. These can then be used to find the work done by the losses in the chain, which in turn is used to find the power losses and chain efficiency.

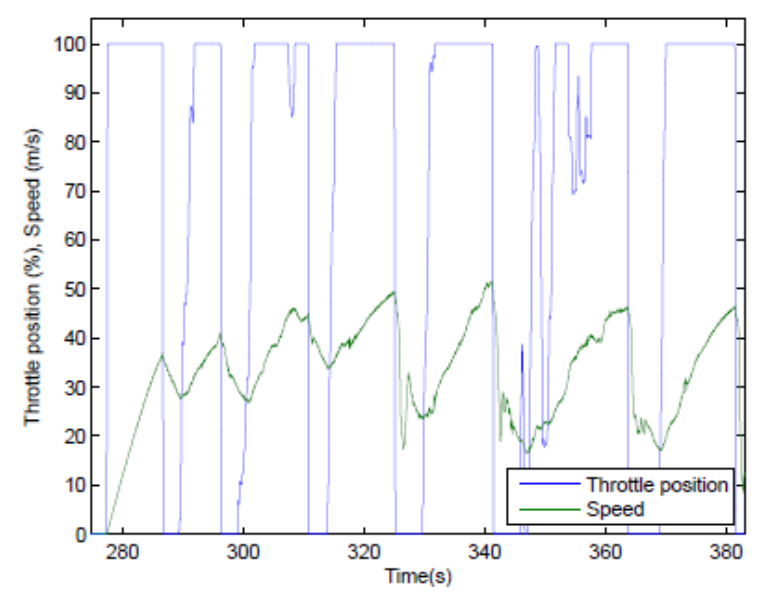

Fig. 3. Typical throttle position input to the motor drive controller and vehicle speed for a racing electric superbike

The chain efficiency is therefore a function of several variables including the torque, speed and dimensions of both the chain and sprockets. It can be seen from race drive-cycle data that the rider throttle application is primarily maximum or zero, as shown Fig 3, so it is a reasonable approximation to use the peak desired wheel torque in the efficiency calculation. It is expected that the bike will require top speeds in the region of 60 to $95 \mathrm{~m} / \mathrm{s}$, leading to an overall required ratio of $3: 7<\mathrm{N} 1 \mathrm{~N} 2$ $<5: 8$ for the chosen motor, if only a chain drive is employed, the system chosen for the University of Nottingham electric superbike. The rather binary throttle input is not typical of a road-bike, and therefore the assumptions used here may not be valid for more general purpose designs. 
Using this design methodology the superbike has achieved impressive results in terms of the power produced at the rear wheel. Fig. 4 shows the typical results in terms of torque and speed for the 2017 drivetrain design, giving a performance well above a standard superbike setup using a conventional internal combustion engine. This performance curve clearly shows the roll-off of the maximum torque above the base speed of the motor where field weakening is used.

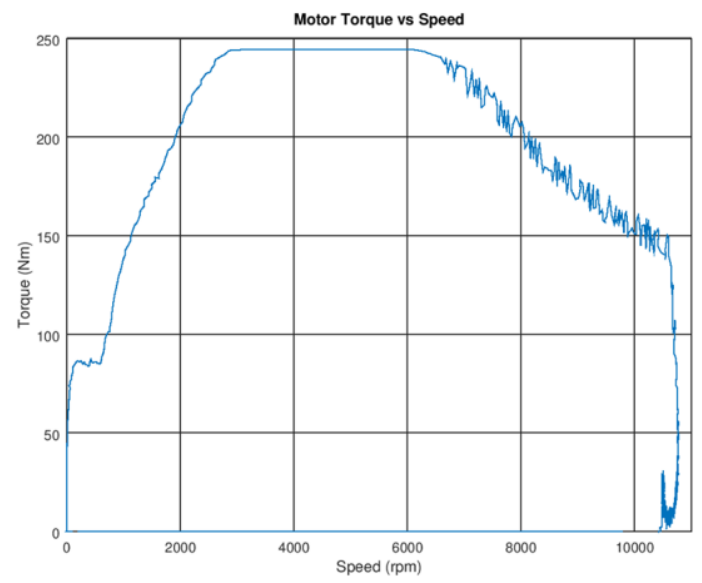

Fig. 4. Typical Torque/Speed Curve measures at the rear wheel based on the 2017 University of Nottingham superbike.

\section{B. Battery Selection}

Selecting the batteries requires choosing a suitable compromise between the required stored energy and power densities. Predictions of the average power requirements were made, from which the total stored energy can be found. This is then split into an appropriate number of series and parallel cells to match the ratings of the chosen motor as well as respecting the maximum voltage allowed by the relevant competitions. Initially, cells with an energy density of $140 \mathrm{Wh} / \mathrm{kg}$ and a discharge rating of $40 \mathrm{C}$ were chosen, however as the power requirements became clearer through analysing recorded race data, the discharge rating has been sacrificed to give a better energy density, enabling the incorporation of a larger battery pack for the same vehicle weight. When optimising the battery for any vehicle the temperature rise of the pack must also be respected as the weight and volume of a forced cooling system (as used on some cars) cannot be accommodated on this type of platform.

\section{Power converter}

The power converter should be designed to match the current and voltage ratings of the motor, with particular consideration given to the need for a high power density without a high lifetime. For the 2016 season the University of Nottingham race team choose Hybridpack devices from Infineon to meet these requirements. These devices have ratings of $800 \mathrm{~A}$ and $650 \mathrm{~V}$ in a compact water-cooled package designed for automotive applications [8]. For reasons of expediency these were initially used with a development kit, later replaced with a drive and control system of our own design. This approach gives great flexibility of the response and set-up of the electrical system to match the particular conditions encountered in racing, without being restricted to changing only the available parameters in commercial software.

\section{Performance Predictions}

Once the main power-train components were identified, comparisons were made with commercial ICE driven bikes, to show compare their performance. The torque available at the rear wheel of each bike is compared over a range of speeds. For the electric bikes, this is simply obtained by multiplying the motor torque-vs speed curve by the chosen gear ratio, as the gear ratio is fixed. The ICE bikes include a gearbox which has to be considered in these calculations. This comparison considers the Kawaski H2r 2004, Suzuki GSXR1000 Nottingham, 2015 Nottingham race bike and the 2016 Nottingham race bike.

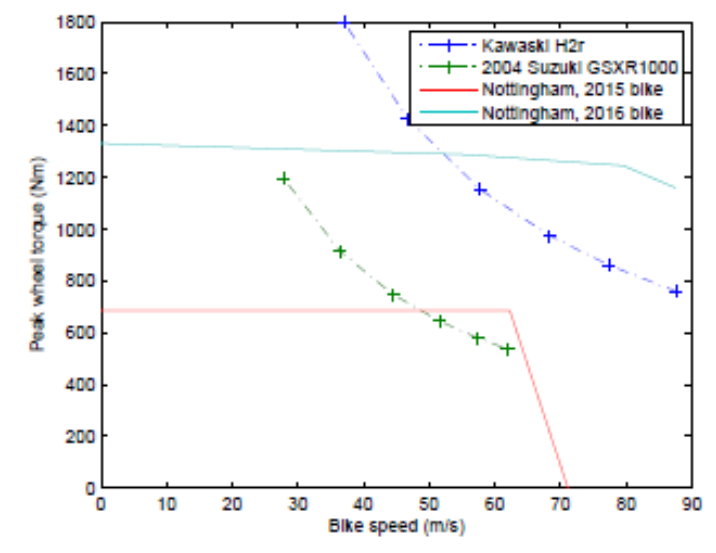

Fig. 5. Comparing the wheel torque of the University of Nottingham electric bikes verses two popular ICE driven bikes at the peak power point for each standard gear ratio.

The overall drive ratios for each gear are calculated [9] [10], with the peak engine torque entered to find the peak torque available in each gear. Figure 3 shows that the wheel torques available are comparable, with the electric bikes giving more or less torque depending on the speed. It is important to note that the points for the ICE bikes are the peak engine torque, so between these values the available torque and thus acceleration is reduced. The top speed of each bike will depend mainly on their maximum power (for a similar drag coefficient) so a useful performance metric is predicting the acceleration to account for differences in performance caused by the additional mass of the electric bikes.

This predicted performance is shown in Fig. 5, where the air resistance and other resistive forces are neglected so the acceleration shown is effectively what could be obtained from zero speed. This analysis implies the acceleration for the ICE bikes in lower gears will be greater than the university 2016 bike, however this does not consider the torque limits imposed by tyre traction and wheel to ground contact. According to [11], this will limit the feasible acceleration to around $10 \mathrm{~ms}^{2}$, with the exact value a function of weight distribution and roadtyre interface friction co-efficient. The values of acceleration above this level are therefore arguably of little use to the rider. 

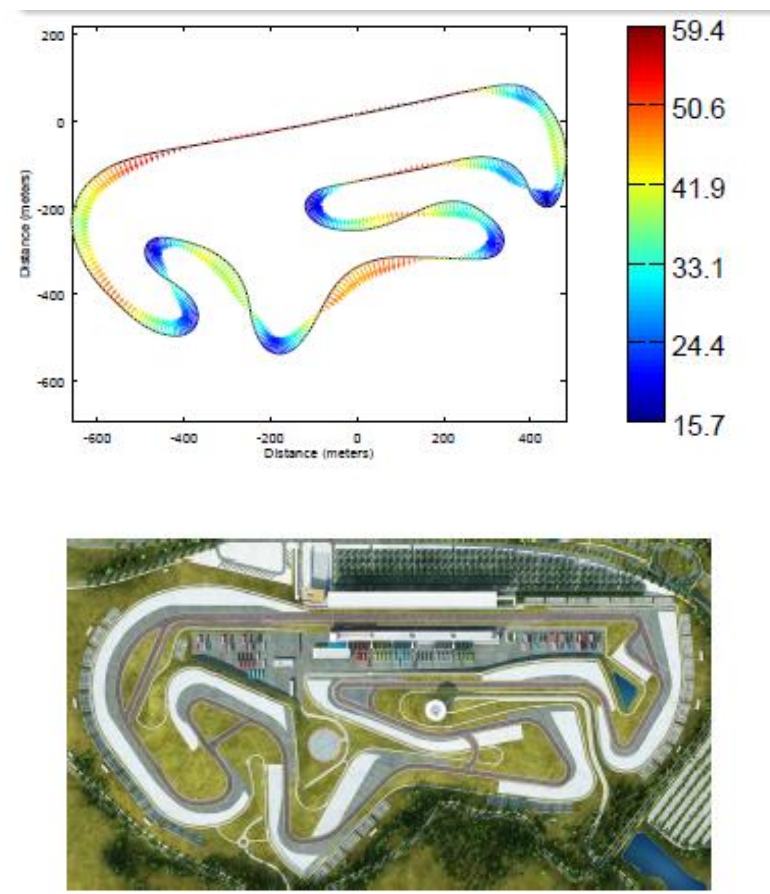

Fig. 6. Speed and Lean Angle of the bike overlaid on a course map of the Portimao Circuit in Portugal

\section{SIMULATION AND VALIDATION}

The model of the bike has been made with the assumption that the rider applies full throttle out of every corner and brakes to a given speed at each corner apex. The drag was calibrated to match the measured total torque requirement when the bike is travelling at a constant, high, speed. All other parameters, such as the mass of the bike and gear ratio are derived from direct measurement.

Fig. 7 compares the simulated bike versus the recorded race data near the beginning of the TT-Zero race. A close match is observed where the throttle input assumption is valid, with some discrepancy when the rider uses partial throttle. Furthermore, when the simulation is applied to the whole lap, the lap time predicted is within $2 \%$ of the actual lap time obtained.

The model will be further verified through application to other races in which the bike competes. By adjusting parameters in the bike, including weight, sprocket sizes and the motor torque-speed characteristic, the lap times and energy usage can be compared with the model. The model can also output detailed information of the energy usage of different parts in the system, which will be used to optimise riding style for improving energy management whilst maintaining or decreasing the lap time.

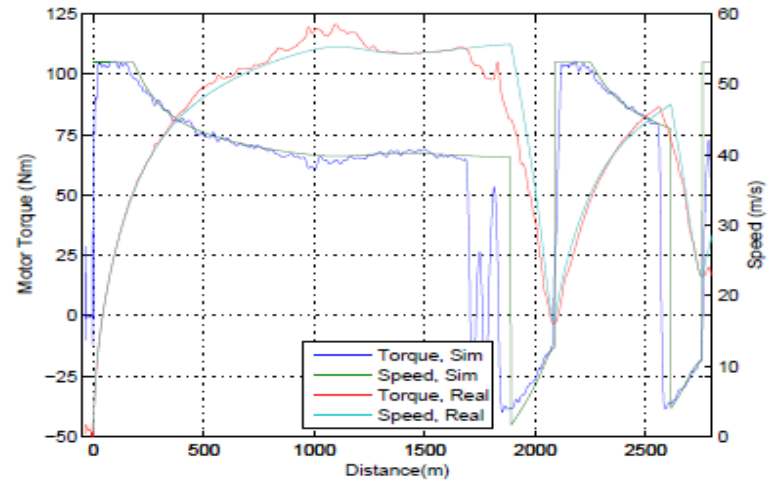

Fig. 7a. Torque and Bike Speed - Simulation and Measurement from TT Zero

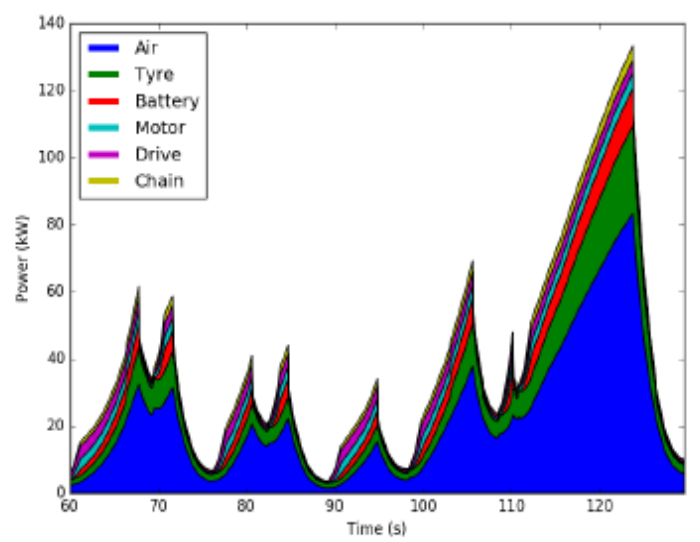

Fig. 7b. Power Losses - Predicted Performance

\section{CONCLUSIONS}

The motorcycle drive system described has been demonstrated as a successful design, through winning the majority of the races it has entered. Although the performance demonstrated so far is still lacking in comparison to ICE counterparts, the bike has not yet been run with the full potential of the electrical drive system, due to limitations in the motor drive which are currently being addressed. The simulation presented shows promise in giving accurate predictions of the lap times that can be obtained for a given racing bike drive-train specification.

The electric superbike has certainly been very successful in attracting interest to the technology and demonstrating that electric motorbikes can be raced at high speeds on demanding tracks. This is an essential part of demonstrating that electric vehicles of the future will be fun and exciting to drive as well as environmentally friendly. 


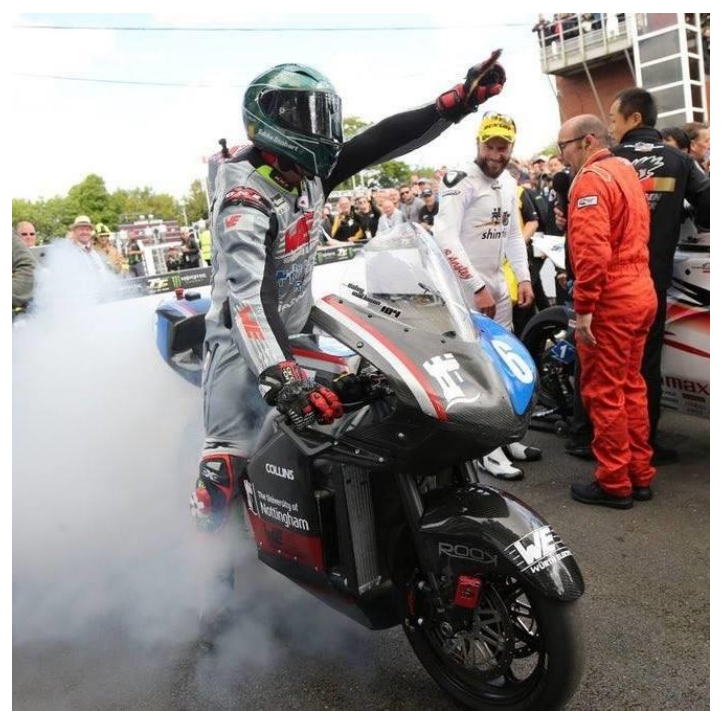

Fig 8. The University of Nottingham Electric Superbike after winning a podium position in the 2017 Ilse of Man TT Zero

\section{ACKNOWLEDGMENT}

The authors would like to thank members of the team for giving up their free time to this project and the riders, Jeremiah Johnson and Daley Mathison for their insight and spirited riding.

\section{REFERENCES}

[1] T. Sarolea. "Sarolea SP7 debuts ahead of the tt zero", 2017 [Online]. http://www.autoblog.com/2015/04/08/2015-saroleasp7- debut-electricmotorcycle/

[2] P. H. Corporation, "Introducing team Parker Brammo", 2015

[3] D. Crolla, "Encyclopedia of Automotive Engineering". Wiley, 2015.

[4] T. Foale, "Motorcycle Handling and Chassis Design: The Art and Science. Tony Foale", 2006.

[5] P. H. Corporation, "GVM series motor datasheet," 2014.

[6] S. Burgess and C. Lodge, "Optimisation of the chain drive system on sports motorcycles," in Sports Engineering, vol. 7. Springer-Verlag, 2004, pp. 65-73.

[7] N. Anderson, "Spur-gear-system efficiency at part and full load," 1980.

[8] I. T. AG, "Evaluation kit for applications with hybridpack2 module," Infineon, Tech. Rep., 2014.

[9] [10] Steve, "Steve's GSXR pages", 2016 [Online].:
http://wotid.com/gsxr/content/view/20/35/

[10] K. M. E. N.V., “ Ninja h2r specifications”, 2016. [Online]. http://www.kawasaki.eu/

[11] V. Cossalter, "Motorcycle Dynamics", LULU, 2006.

[12] I. D. of Tourism, "Official TT Guide”, Mannin Media, 1992.

[13] H. B. Ahmad RAHMOUN, "Modelling of Li-ion batteries using equivalent circuit diagrams," in Electrical review, 2008, pp. 152-156.

[14] M. P. Duan Graovac, "IGBT power losses calculation using the datasheet parameters," Infineon, Tech. Rep., 2009.

[15] H. G. Ltd., "HPC gears technical index”, 2010 